\title{
Prevalência e fatores condicionantes do aleitamento materno - Estudo ALMAT
}

Sílvio Barge,* Marisa Carvalho**

\section{RESUMO}

Objetivos: Determinar a prevalência do Aleitamento Materno (AM) aos 6 meses e verificar se o seu abandono precoce se relaciona com a idade materna, paridade, escolaridade, situação profissional, tabagismo, alcoolismo e nível de conhecimento das mães sobre os benefícios da amamentação.

Tipo de Estudo: Estudo observacional transversal analítico.

Local: Unidade de Saúde Familiar (USF) Lethes e USF Mais Saúde, da Unidade Local de Saúde do Alto Minho.

População: Parturientes de 1 de janeiro a 31 de dezembro de 2009 inscritas nas USF referidas.

Métodos: Entre 1 de setembro e 15 de outubro de 2010, foi realizada uma entrevista telefónica a 163 parturientes (106 da USF Lethes e 57 da USF Mais Saúde) selecionadas de forma aleatória estratificada proporcional, a partir de uma listagem das parturientes do ano de 2009 dada pelo programa informático SINUS.

A informação foi recolhida através da aplicação de um questionário elaborado pelos autores. Foram determinados resultados referentes à estatística descritiva e inferencial.

Resultados: A prevalência estimada do AM aos 6 meses foi de 36\% (IC 95\%, 29\% - 43\%). Em média, o AM foi mantido durante 5 meses. A prevalência foi maior nas parturientes com maior idade. Verificou-se ainda uma maior prevalência de AM nas parturientes com baixa escolaridade e licenciadas, e menor nas parturientes que frequentaram as aulas de preparação para o parto, mas, na análise estratificada, constatou-se que a idade atuou como variável de confundimento, não se verificando associação estatisticamente significativa para estas variáveis.

Conclusões: A prevalência do AM aos 6 meses foi semelhante à dos estudos existentes, mas inferior às metas estabelecidas pela OMS. A associação entre o AM e a idade está em concordância com a literatura. Consideramos que este estudo é original em Portugal na análise de alguns fatores condicionantes da prevalência do AM aos 6 meses, no âmbito dos cuidados primários.

Palavras-chave: Aleitamento Materno; Prevalência.

\section{INTRODUÇÃO}

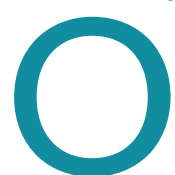

Aleitamento Materno (AM) é o principal meio de nutrição durante o primeiro ano de vida, nomeadamente no primeiro semestre de vida. ${ }^{1}$ Várias organizações, nomeadamente a Organização Mundial de Saúde (OMS) em conjunto com o Fundo das Nações Unidas para a

*Interno do internato médico de Medicina Geral e Familiar. USF Lethes -ACES Alto Minho.

**Interna do internato médico de Medicina Geral e Familiar. USF Mais Saúde - ACES Alto Minho.

Texto escrito conforme o Acordo Ortográfico
Infância (UNICEF), a Academia Americana de Pediatria e a Sociedade Canadiana de Pediatria, recomendam AM exclusivo até aos 6 meses e a sua manutenção, se possível, até aos dois anos. ${ }^{2-4}$

Todavia, apesar da natural disponibilidade do AM e das suas vantagens, diversos fatores influenciam a sua implementação e respetivo abandono precoce. ${ }^{3,5}$

A amamentação proporciona vantagens não apenas em termos de crescimento, mas também no desenvolvimento neurológico e emocional do ser humano. ${ }^{6} \mathrm{O}$ leite materno diminui o risco de diversas patologias agudas e crónicas para o recém-nascido, tais como pro- 
teção imunológica, com consequente diminuição do risco de otite, meningite e diarreia, diminuição do risco de desenvolvimento de alergias, doença celíaca, doença inflamatória intestinal, diabetes tipo 1 e 2, hipertensão arterial, dislipidemia e, possivelmente, alguns tipos de cancro. ${ }^{1,3,7} \mathrm{O}$ AM apresenta também diversas vantagens para a mãe, nomeadamente involução uterina mais rápida e completa, contraceção natural, recuperação de peso ideal mais rápida, diminuição do risco de neoplasias (mama e ovário) e de osteoporose. ${ }^{1,3,7,8} \mathrm{O}$ AM materno é ainda importante no estabelecimento de um forte vínculo emocional entre a mãe e o bebé. ${ }^{1,3,7,8}$

O AM depende de diversos fatores socioculturais, profissionais, nível educacional e de ações de promoção da saúde levadas a cabo por profissionais de saúde empenhados e motivados. ${ }^{5-17}$

Diversos estudos realizados em Portugal apontam para uma alta taxa de abandono da amamentação, estimando-se que apenas cerca de um terço das parturientes (entre $22,4 \%$ e $52,8 \%$ ) continua a amamentar aos seis meses após o parto. ${ }^{10-17} \mathrm{O}$ conhecimento desta prevalência na nossa população, bem como o de possíveis causas para o abandono do AM, poderá ser um ponto de partida para a implementação de medidas que visem uma melhor e mais eficaz promoção do aleitamento materno na nossa população.

Neste contexto, o objetivo deste estudo consistiu em determinar a prevalência do AM aos 6 meses em cuidados primários, bem como verificar se o seu abandono precoce é independente da idade, paridade, escolaridade, situação profissional, tabagismo, alcoolismo e nível de conhecimento das mães sobre os benefícios da amamentação.

\section{MÉTODOS}

Realizou-se um estudo observacional analítico transversal, que decorreu de 1 de agosto de 2010 a 30 de novembro de 2010 (duração de 4 meses).

A recolha de dados foi realizada por entrevista telefónica em duas Unidades de Saúde Familiar (USF) do concelho de Ponte de Lima: USF Lethes e USF Mais Saúde. Estas USF's são simultaneamente os locais de formação dos dois investigadores que realizaram o estudo.

A população do estudo correspondeu às parturien- tes de 1 de janeiro de 2009 a 31 de dezembro de 2009 inscritas nas USF's acima referidas. Foram excluídas as parturientes que por razões médicas (seropositividade para o Vírus da Imunodeficiência Humana (VIH), seropositividade para o Vírus T-Linfotrópico Humano (HTLV-1), tuberculose ativa não tratada, quimioterapia, consumo de fármacos contraindicados durante a amamentação, consumo de drogas ilícitas, seropositividade para citomegalovírus em mães com recém-nascidos prematuros, doença metabólica do recém-nascido, como galactosémia e, lesões herpéticas ativas na mama $)^{5,19}$ não puderam amamentar.

A amostragem foi efetuada por técnica aleatória estratificada proporcional.

O número total de parturientes foi obtido a partir da listagem dos nascimentos registados durante o ano de 2009, utilizando o programa SINUS. Verificou-se um total de 222 nascimentos inscritos nas USF's, dos quais seis eram gémeos e seis parturientes apresentavam critérios de exclusão. Assim a população foi de 210 parturientes (137 da USF Lethes e 73 da USF Mais Saúde). Considerando um nível de precisão de $5 \%$ e um intervalo de confiança de $95 \%$, com uma prevalência esperada de AM aos 6 meses de $34 \%,{ }^{12,13,16,17}$ calculou-se uma dimensão da amostra de 131 indivíduos, utilizando o programa EpiInfo versão 3.5. Prevendo-se uma taxa de resposta de 80\%, a amostra foi alargada para 163 (106 da USF Lethes e 57 da USF Mais Saúde).

Após o cálculo da amostra, a seleção das parturientes a ser inquiridas foi efetuada por técnica aleatória simples, recorrendo ao programa informático disponível no sítio da internet http://www.random.org/integers/.

As variáveis estudadas foram o AM aos 6 meses (definida como sim, se aos seis meses de idade do recém-nascido a mãe mantinha pelo menos uma mamada por dia), variáveis sociodemográficas (idade, escolaridade, paridade), alcoolismo (considerado presente se a mãe ingeriu duas ou mais bebidas por dia, durante os primeiros seis meses após o parto), tabagismo (considerado presente se a mãe consumiu diariamente tabaco durante a gravidez e/ou nos primeiros seis meses após o parto), frequência das aulas de preparação para o parto (definido como sim, caso tenha frequentado pelo menos três aulas de preparação para o parto) e vigilância na USF (definido como sim, caso tenham sido 
realizadas pelo menos seis consultas de saúde materna na USF).

Para a recolha de dados foi aplicado um questionário elaborado pelos investigadores (Anexo I).

Para obtermos uma maior taxa de resposta e reduzir o tempo de recolha de dados, o questionário foi aplicado através de entrevista telefónica. Aquando do contacto telefónico, foi obtido, oralmente, o consentimento informado.

O contacto telefónico foi realizado até cinco tentativas em diferentes dias e horários para cada parturiente selecionada. As parturientes às quais não foi possível efetuar a recolha dos dados por não terem contacto telefónico no processo ou continuarem incontactáveis após as cinco tentativas de contacto foram consideradas como não respondentes, assim como aquelas que, após adequada explicação do estudo, se recusaram a participar.

Os investigadores realizaram um treino conjunto para uniformizar a entrevista, tendo realizado um teste piloto a 20 indivíduos da população de estudo, que não pertenciam à amostra.

Os dados recolhidos foram codificados, garantindose a confidencialidade, e registados numa base de dados informática usando o programa SPSS ${ }^{\circledast}$ versão 17.0, que também foi utilizado para o tratamento estatístico dos mesmos.

Procedeu-se à análise descritiva, à análise bivariada através dos testes Qui-quadrado e de Fisher (para comparação de proporções) e Mann-Witney (para comparação de médias) e à análise multivariada utilizando a Regressão Logística. Nas variáveis em que se obteve uma relação estatisticamente significativa foi, ainda, calculada a razão de prevalência, dividindo a taxa de prevalência de AM aos 6 meses no grupo de puérperas que estava presente a variável em estudo pela taxa de prevalência de $\mathrm{AM}$ aos 6 meses no grupo que essa mesma variável não estava presente.

O nível de significância adotado para aceitação de diferenças estatisticamente significativas foi de 0,05 .

O estudo foi aprovado pela comissão de ética da Unidade Local de Saúde do Alto Minho e a sua realização foi autorizada pela respetiva administração.

\section{RESULTADOS}

Responderam ao questionário 158 parturientes, o que corresponde a uma taxa de resposta de $96,9 \%$. A idade das inquiridas variou entre 19 e 44 anos, com uma média de $29( \pm 5)$ anos. A maioria possuía entre 7 a 12 anos de escolaridade e cerca de metade era ativa. A maioria foi vigiada na USF, mas apenas $41 \%$ frequentou as aulas de preparação para o parto. As caraterísticas sociodemográficas das parturientes estão resumidas no Quadro I.

A prevalência de AM aos 6 meses foi de 36\% (IC 95\% [29-42]). A prevalência de AM aos 3, 9 e 12 meses foi, respetivamente, $60 \%, 27 \%$ e $14 \%$.

Obteve-se uma relação estatisticamente significativa entre o AM aos 6 meses e a idade materna (aumento da prevalência do AM com a idade), a escolaridade (prevalência de AM maior nos extremos de escolaridade, com razão de prevalência de 1,59 (IC 95\% [0,942,68]), 1,00 (IC 95\% [0,55-1,82]), 0,71 (IC 95\% [0,38-1,30]) e 1,32 (IC 95\% [0,69-2,51]), respetivamente para $\leq 6$ anos, 7 a 9 anos, 10 a 12 anos e mais de 12 anos de escolaridade), e a frequência de aulas de preparação para o parto (prevalência do AM maior nas mulheres que não frequentaram as aulas de preparação para o parto, com razão de prevalência de 0,56 (IC 95\% [0,34-0,91]).

\begin{tabular}{|c|c|c|c|}
\hline & & $\mathrm{n}$ & $\%$ \\
\hline Idade mãe & [anos] (média $\pm D P$ ) & & \\
\hline \multirow[t]{4}{*}{ Escolaridade } & $\leq 6$ anos & 35 & $22 \%$ \\
\hline & 7-9 anos & 41 & $26 \%$ \\
\hline & 10-12 anos & 62 & $39 \%$ \\
\hline & $>12$ anos & 20 & $13 \%$ \\
\hline Situação profissional & ativa & 84 & $53 \%$ \\
\hline Tabagismo & $\operatorname{sim}$ & 7 & $4 \%$ \\
\hline Alcoolismo & $\operatorname{sim}$ & 1 & $1 \%$ \\
\hline \multirow[t]{3}{*}{ N. ${ }^{\circ}$ partos } & 1 & 81 & $51 \%$ \\
\hline & 2 & 66 & $42 \%$ \\
\hline & $\geq 3$ & 11 & $7 \%$ \\
\hline Vigilância na USF & $\operatorname{sim}$ & 141 & $89 \%$ \\
\hline APP & $\operatorname{sim}$ & 65 & $41 \%$ \\
\hline
\end{tabular}

APP: Aulas de Preparação para o Parto, USF: Unidade de Saúde Familiar 
Não se encontrou associação estatisticamente significativa entre o AM aos 6 meses e a situação profissional, o tabagismo, o alcoolismo e a vigilância na USF. Apesar de não se ter obtido relação estatisticamente significativa ( $\mathrm{p}=0,36$ ), observou-se uma tendência para um aumento do AM aos seis meses com o aumento da paridade.

Para avaliar a possibilidade de existência de fatores de confundimento realizou-se uma análise multivariada por regressão logística, deixando de haver relação estatisticamente significativa relativamente aos fatores escolaridade e frequência das aulas de preparação para o parto.

Calculou-se, ainda, a prevalência de AM aos seis meses em cada uma das USF's e, ao contrário do esperado obteve-se uma diferença de prevalências estatisticamente significativa, tendo sido de $29 \%$ numa das USF's e $48 \%$ na outra $(\mathrm{p}=0,019)$. Fomos então verificar se as populações eram idênticas relativamente a todos os fatores estudados e verificou-se que estas diferiam, de forma estatisticamente significativa, a nível da escolaridade $(p=0,008)$ e da paridade $(p=0,019)$. No entanto, como referido anteriormente, neste estudo não existiu relação estatisticamente significativa entre estes fatores e o AM aos 6 meses.

\section{DISCUSSÃO}

Neste estudo a prevalência estimada do AM aos seis meses foi de $36 \%$ $( \pm 7)$, o que está de acordo com os resultados obtidos por estudos nacionais e internacionais. ${ }^{2,11-13,15-17,19}$

Neste estudo foi encontrada uma relação positiva estatisticamente significativa entre a idade materna e o AM aos 6 meses. Esta associação está de acordo com a literatura. ${ }^{10,12,13}$ Verificouse também uma associação positiva entre o AM aos 6 meses e os extremos de escolaridade (menor ou igual a 6 anos de escolaridade e ensino superior) e associação negativa entre o AM aos 6 meses e a frequência nas aulas de preparação para o parto. Apesar de não ter sido encontrada uma diferença estatisticamente significativa entre o AM aos 6 meses e a maior paridade, observou-se uma tendência positiva entre a maior paridade e a maior prevalência de AM. Todavia, após a análise multivariada, tais associações deixaram de se verificar, pois a variável idade constituiu uma variável de confundimento. As parturientes nos extremos de escolaridade e as parturientes multíparas tinham uma média de idade superior e, por outro lado, as parturientes frequentadoras das aulas de preparação para o parto tinham uma média de idade inferior. Existem trabalhos publicados que reportam existir uma associação positiva entre o AM e a maior escolaridade $^{11-14,17}$ e paridade, ${ }^{10,11,13,16,17}$ o que está em desacordo com os nossos resultados. No entanto, nenhum

\begin{tabular}{|c|c|c|c|c|}
\hline \multirow{2}{*}{\multicolumn{5}{|c|}{$\begin{array}{l}\text { QUADRO II. Análise bivariada do score de aleitamento ma } \\
\text { meses com as variáveis sociodemográficas estudadas } \\
\text { AM aos } 6 \text { meses }\end{array}$}} \\
\hline & & & & \\
\hline & \multicolumn{2}{|c|}{$\begin{array}{r}\text { AMM aos 6 mes } \\
\text { Não }[\mathrm{n}(\%)] \\
\end{array}$} & \multirow{2}{*}{$\begin{array}{c}\operatorname{Sim}[n(\%)] \\
32 \pm 4\end{array}$} & \multirow{2}{*}{$\frac{P}{<0,001^{*}}$} \\
\hline Idade mãe & [anos] (média $\pm D P$ ) & $28 \pm 5$ & & \\
\hline \multirow[t]{4}{*}{ Escolaridade } & $\leq 6$ anos & $16 \quad(46 \%)$ & 19 (54\%) & $0,022^{¥}$ \\
\hline & 7-9 anos & $27 \quad(66 \%)$ & $14(34 \%)$ & \\
\hline & 10-12 anos & $47 \quad(76 \%)$ & 15 (24\%) & \\
\hline & $>12$ anos & 11 (55\%) & $9(45 \%)$ & \\
\hline \multirow[t]{2}{*}{ Situação profissional } & ativa & $49 \quad(58 \%)$ & $35(42 \%)$ & $0,119^{¥}$ \\
\hline & inativa & $52 \quad(70 \%)$ & $22(30 \%)$ & \\
\hline \multirow[t]{2}{*}{ Tabagismo } & $\operatorname{sim}$ & $6 \quad(86 \%)$ & 1 (14\%) & $0,423^{\ddagger}$ \\
\hline & não & $95 \quad(63 \%)$ & $56(37 \%)$ & \\
\hline \multirow[t]{2}{*}{ Alcoolismo } & $\operatorname{sim}$ & $1(100 \%)$ & $0 \quad(0 \%)$ & $1,0^{\ddagger}$ \\
\hline & não & 100 (64\%) & $57(36 \%)$ & \\
\hline \multirow[t]{3}{*}{ N. ${ }^{\circ}$ partos } & 1 & $56 \quad(69 \%)$ & $25(31 \%)$ & $0,360^{\neq}$ \\
\hline & 2 & $39 \quad(59 \%)$ & $27(41 \%)$ & \\
\hline & $\geq 3$ & $6 \quad(55 \%)$ & $5(45 \%)$ & \\
\hline \multirow[t]{2}{*}{ Vigilância na USF } & $\operatorname{sim}$ & $87 \quad(82 \%)$ & $54(18 \%)$ & $0,094^{¥}$ \\
\hline & não & $14 \quad(62 \%)$ & $3(38 \%)$ & \\
\hline \multirow[t]{2}{*}{ APP } & $\operatorname{sim}$ & $49 \quad(75 \%)$ & $16(25 \%)$ & $0,012^{\ddagger}$ \\
\hline & não & $52 \quad(56 \%)$ & $41(44 \%)$ & \\
\hline
\end{tabular}

APP: Aulas de Preparação para o Parto, USF: Unidade de Saúde Familiar, *Teste de Mann-Whitney, ${ }^{*}$ Teste do Qui-quadrado, ${ }^{\ddagger}$ Teste de Fisher 


\begin{tabular}{|c|c|c|c|}
\hline \multicolumn{4}{|c|}{$\begin{array}{l}\text { QUADRO III. Análise multivariada dos fatores associa- } \\
\text { dos ao aleitamento materno aos } 6 \text { meses }\end{array}$} \\
\hline \multicolumn{2}{|l|}{ Variável } & \multicolumn{2}{|c|}{ AM aos 6 meses } \\
\hline & & OR & $p$ \\
\hline Idade & & & $<0,001$ \\
\hline \multirow[t]{4}{*}{ Escolaridade } & $\leq 6$ anos & 1 & 0,402 \\
\hline & 7-9 anos & 0,44 & \\
\hline & 10-12 anos & 0,27 & \\
\hline & $>12$ anos & 0,69 & \\
\hline \multirow[t]{2}{*}{ Frequência APP } & $\operatorname{sim}$ & 1 & 0,111 \\
\hline & não & 2,41 & \\
\hline \multirow[t]{2}{*}{ USF } & 1 & 1 & 0,047 \\
\hline & 2 & 2,23 & \\
\hline
\end{tabular}

APP: Aulas de Preparação para o Parto, USF: Unidade de Saúde Familiar

dos estudos referidos refere que tenha sido efetuada análise multivariada para excluir a existência de fatores de confundimento, que poderão ter existido, tal como aconteceu na nossa população. Mais estudos, que utilizem amostras maiores, idealmente multicêntricos e, em que seja analisada a possibilidade da existência de fatores de confundimento deverem ser realizados para esclarecer tais discrepâncias.

Não foi obtida associação estatisticamente significativa entre o AM e a vigilância médica em consultas de saúde materna da USF, nem com a frequência de aulas de preparação para o parto. Na nossa opinião, tais resultados poderão ter sido resultado da contaminação exterior com informações e atitudes promotoras do AM, veiculadas pela comunicação social, por outros profissionais de saúde externos às USF's, por familiares, amigos e por sites de informação via internet, anulando desta forma os efeitos supostamente benéficos da intervenção pelos profissionais de saúde das USF's. ${ }^{11,17}$

A análise inferencial das variáveis tabagismo e consumo de álcool não revelou qualquer associação estatisticamente significativa com o AM aos 6 meses, ao contrário da literatura. ${ }^{17}$ É de salientar que nesta análise foi necessário aplicar um teste estatístico com menor poder (Teste de Fisher), havendo a possibilidade de ter sido introduzido um erro aleatório do tipo II.
Procedeu-se à análise estratificada por USF das parturientes. Constatou-se haver uniformidade entre os dois grupos quanto à idade. Para surpresa dos investigadores deste estudo, obteve-se uma diferença estatisticamente significativa na prevalência do AM aos seis meses entre as duas USF. Foi, então, estudada a influência dos fatores, separadamente nas duas USF's e verificou-se que numa das USF's a menor e maior escolaridade está associada a aumento da prevalência do $\mathrm{AM}$ aos 6 meses, enquanto na outra USF tal não se verifica. Assim, os diferentes resultados de prevalência encontrados entre as USF's poderão ter-se devido a influência de outros fatores não caraterizados neste estudo, tais como o seguimento em consultas médicas particulares de obstetrícia, estado civil, raça, nível socioeconómico e diferentes metodologias de trabalho entre as duas equipas de saúde, nomeadamente no que concerne à promoção do $\mathrm{AM}$, que poderão ter atenuado a influência da escolaridade na USF em que não se verificou associação com essa variável.

O presente estudo apresenta algumas limitações. A população estudada apresenta dimensão reduzida, pelo que a estimativa da prevalência obtida encontra-se num intervalo relativamente amplo. $O$ reduzido tamanho amostral poderá, ainda, ter impedido a rejeição de algumas das hipóteses nulas, quando na realidade poderão ser falsas. A utilização de um questionário não validado poderá ter introduzido um viés de medição. Os autores procuraram minimizar este inconveniente, utilizando perguntas concisas, claras e de respostas objetivas. A existência de dois entrevistadores poderá ter condicionado um viés do entrevistador por diferenças na recolha de dados, todavia, este viés foi minimizado pelo treino conjunto para uniformização da entrevista e pela realização do teste piloto. A recolha de dados implicou um esforço de memória por parte dos participantes no estudo, pelo que poderá ter sido introduzido um viés de memória. Procurou-se minimizar este viés através da realização de uma entrevista pausada e da aplicação de um questionário pouco extenso, com perguntas simples, de forma a facilitar a cooperação dos participantes e a recordação dos factos relevantes para o estudo.

Tanto quanto os investigadores deste trabalho têm conhecimento, tratou-se de um estudo original em Portugal, no âmbito dos cuidados primários, uma vez que 
a maioria dos estudos existentes é feita a nível hospitalar, por pediatras..$^{10,12,14-17}$ De referir que os poucos estudos efetuados a nível dos cuidados primários ${ }^{11,13}$ utilizaram amostras de menor dimensão que a nossa e não foi efetuada, ou pelo menos não se encontra descrita, a realização de análise multivariada. De salientar, ainda, deste trabalho que foram estudados fatores de intervenção profissionais potencialmente promotores do $\mathrm{AM}^{20}$ levadas a cabo pelas equipas de saúde das USF's (vigilância da gravidez na USF e frequência de aulas de preparação para o parto) que, no entanto, como se verificou no estudo, não parecem ser muito influentes na manutenção do AM.

Os autores salientam que o estudo foi realizado numa população de uma zona geográfica de Portugal predominantemente rural, com características socioculturais específicas e pertencentes a duas USF's, com rotinas de trabalho próprias, pelo que, apesar da amostra ser representativa da população de parturientes inscritas nestas duas USF's, não é possível generalizar os resultados para a população portuguesa, nem tão pouco para o distrito de Viana do Castelo.

Nas metas para a saúde no ano de 2000 a Organização Mundial de Saúde definiu como objetivos aumentar a prevalência do AM aos 6 meses para $50 \% .^{21} \mathrm{~A}$ prevalência do AM encontrada expressa uma realidade negativa, onde apenas cerca de um terço das parturientes continuam a amamentar (exclusivamente ou não) aos 6 meses. Tendo em conta os benefícios do AM até pelo menos aos 6 meses de idade e o seu impacto potencial na melhoria dos cuidados de saúde primários na nossa população, importa conhecer melhor os fatores condicionantes do AM em cuidados primários. Os resultados obtidos levam-nos a pensar que os motivos de abandono do AM não podem ser encarados numa perspetiva simplista, mas sim num contexto multifactorial complexo, que deve ser averiguado através da realização de mais estudos, mais abrangentes e de maior dimensão.

É importante divulgar estes resultados na comunidade médica, como um incentivo a novos estudos e à implementação e reforço de medidas promotoras do AM.

\section{REFERÊNCIAS BIBLIOGRÁFICAS}

1. World Health Organization. Infant and young child feeding counselling: an integrated course. Geneva: WHO; 2006. Disponível em: http://whqlibdoc.who.int/publications/2006/9789241594776_eng.pdf [acedido em 16/08/2010].

2. World Health Organization/UNICEF. Global strategy for infant and young child feeding. Geneva:WHO; 2003. Disponível em: http://whqlibdoc.who.int/publications/2003/9241562218.pdf [acedido em 16/09/2010].

3. Gartner LM, Morton J, Lawrence RA, Naylor AJ, O'Hare D, Schanler RJ, et al. Breastfeeding and the use of human milk. Pediatrics 2005 Feb; 115 (2): 496-506.

4. Boland M. Exclusive breastfeeding should continue to six months. Paediatr Child Health 2005 Mar; 10 (3): 148.

5. Schanler R J., Potak D C. Breastfeeding: parental education and support. In: UpToDate; 2010. Disponível em http://www.uptodate.com [acedido em 16/08/2010].

6. Schanler R J. Infant benefits of breastfeeding. In: UpToDate; 2010. Disponível em http://www.uptodate.com [acedido em 16/08/2010].

7. Schanler R J. Maternal and economic benefits of breastfeeding. In: UpToDate; 2010. Disponível em: http://www.uptodate.com [acedido em 16/08/2010].

8. Guise JM, Palda V, Westhoff C, Chan BK, Helfand M, et al. The effectiveness of primary care-based interventions to promote breastfeeding: systematic evidence review and meta-analysis for the US Preventive Services Task Force. Ann Fam Med 2003 Jul-Aug; 1: 70-8.

9. Oliveira D, Valente C.Adesão ao aleitamento materno. Nascer e Crescer 1999; 8 (1): 31-3.

10. Marques A, Resende C, Fernandes S, Santos S, Campos J, Faria C, et al. Aleitamento materno no distrito de Viseu entre 1999 e 2009. SaudeInfant 2010; 32 (2): 77-82.

11. Rebimbas S, Pinto C, Pinto R. Aleitamento Materno: Análise da Situação num Meio Semi-urbano. Nascer e Crescer 2010; 19 (2): 68-73.

12. Sarafana S, Abecasis F, Tavares A, Soares I, Gomes A. Aleitamento Materno: evolução na última década. Acta Pediatr Port 2006 Jan-Fev; 37 (1): 9-14.

13. Caldeira T, Moreira P, Pinto E. Aleitamento materno: estudo dos factores relacionados com o seu abandono. Rev Port Clín Geral 2007 NovDec; 23 (6): 685-99.

14. Alves A, Lamy S, Henriques G, Virella D, Carreiro H, Lynce N, et al. Aleitamento materno nos concelhos de Cascais, Amadora e Sintra: Porquê o abandono precoce?. Saude-Infant 1999; 21 (1): 43-50.

15. Rocha L, Gomes A. Prevalência do aleitamento materno nos primeiros seis meses de vida. Saude-Infant; 1998; 20 (3): 59-66.

16. Lopes B, Marques P. Prevalência do aleitamento materno no distrito de Viana do Castelo nos primeiros 6 meses de vida. Rev Port Clín Geral 2004 Set-Out; 20 (5): 539-44.

17. Sandes AR, Nascimento C, Figueira J, Gouveia R, Valente S, Martins S, et al. Aleitamento materno: prevalência e fatores condicionantes. Acta Med Port 2007 Mai-Jun; 20 (3): 193-200.

18. World Health Organization. Aceptable medical reasons for use of breastmilk substitutes. Geneva: WHO; 2009. Disponível em http://whqlibdoc.who.int/hq/2009/WHO_FCH_CAH_09.01_eng.pdf [acedido em 16/08/2010].

19. Statement by WHO director-General Dr Margaret Chan. World breastfeeding Week 2008: going for the gold by supporting mothers to breast- 
feed. Disponível em http://centuryware.net/southasiannewsleader. com/news2pdf.php?sid=8 [acedido em 16/09/2010].

20. Arora S, McJunkin C, Wehrer J, Kuhn P. Major factors influencing breastfeeding rates: mother's perception of father's attitude and milk supply. Pediatrics 2000 Nov; 106 (5): e67.

21. World Health Organization. The optimal duration of exclusive breastfeeding: Report of an expert consultation. Switzerland 28-30 March 2001. Disponível em http://whqlibdoc.who.int/hq/2001/WHO_NHD_ 01.09.pdf [acedido em 16/09/2010].

\author{
ENDEREÇO PARA CORRESPONDÊNCIA \\ Sílvio Anjos Rocha Barge \\ Av. Bombeiros Voluntários, n. ${ }^{\circ} 45$ \\ Ed. S. Miguel, Fração A \\ 4990-344 Feitosa PTL \\ E-mail: silvioanjos80@gmail.com \\ Recebido em 01/06/2011 \\ Aceite para publicação em 14/08/2011
}

Os autores declaram não possuir quaisquer conflitos de interesse.

\section{ABSTRACT}

\section{PREVALENCE OF BREASTFEEDING AND ASSOCIATED FACTORS - ALMAT STUDY}

Objective: To determine the prevalence of breastfeeding at 6 months of age and to determine if the early cessation of breastfeeding is associated with maternal age, parity, education, employment status, smoking, alcohol consumption and knowledge of the benefits of breastfeeding.

Study Type: Cross-sectional study.

Location: USF Mais Saúde and USF Lethes, Local Health Unit of Alto Minho.

Population: Women giving birth in 2009 in these regions.

Methods: Between September 1 and October 15, 2010, we conducted telephone interviews with 163 mothers (106 from USF Lethes and 57 from USF Mais Saúde) selected from a list of the women giving birth in 2009 identified in the electronic medical record. The information was gathered using a questionnaire developed by the authors. The data collected were analyzed using descriptive and inferential statistics.

Results: The prevalence of breastfeeding at 6 months was 36\% (29\% - 43\%, Cl 95\%). The mean duration of breastfeeding was 5 months. The prevalence of breastfeeding was higher in older women. The prevalence of breastfeeding was high in women in lowest and highest level of education. It was lower in women who attended birth preparation classes. In a multivariate analysis, age was found to be a confounding variable, so these variables (education and pre-natal classes) were found not to be associated with breastfeeding.

Conclusions: The prevalence of breastfeeding at 6 months of age in this population was found to be similar to that found in other studies, but lower than the goal by set by the World Health Organization. The association between breastfeeding and age is similar to that found in the literature. We believe that this study contributes to our knowledge of the factors associated with the prevalence of breastfeeding at 6 months of age in Portugal.

Keywords: Breastfeeding; Prevalence. 


\section{ANEXO I \\ QUESTIONÁRIO}

Estudo clínico: Prevalência e fatores condicionantes do Aleitamento Materno

Investigador/N. ${ }^{\circ}$ participante

1. Idade

2. Escolaridade

3. Situação profissional Activo Inactivo

4. Hábitos tabágicos durante a gravidez e/ou primeiros 6 meses após o parto

Sim Não

5. Consumo de bebidas alcoólicas nos primeiros 6 meses após o parto

Sim Não

6. N. ${ }^{\circ}$ de partos
$\begin{array}{llll}1 & 2 & 3 & \geq 4\end{array}$

7. A gravidez foi vigiada na USF?

Sim Não

8. Frequentou aulas de preparação para o parto?

Sim Não

9. Quanto tempo amamentou o seu último filho? meses
Instruções de preenchimento do questionário

- Todas as questões referem-se à sua última gravidez, cujo parto ocorreu em 2009

- Idade - em anos, na altura do parto

- Escolaridade - número de anos de escolaridade completos

- Situação profissional - definida como empregada ou desempregada aos 5 meses pós parto

- Tabagismo - consumo diário de tabaco (pelo menos 1 cigarro por dia) durante a gravidez e/ou os primeiros seis meses após o parto

- Alcoolismo - ingestão maior ou igual a 2 bebidas por dia, durante os primeiros 6 meses após o parto

- Paridade - número de partos após às 28 semanas de gestação

- Frequentou aulas de preparação para o parto - definido como sim, caso tenha frequentado pelo menos 3 aulas de preparação para o parto

- Gravidez vigiada na USF - definido como sim, caso tenha feito pelo menos 6 consultas de Saúde Materna na USF

- Quanto tempo amamentou o seu último filho - definido como intervalo de tempo entre o parto e o momento em que foi interrompida a amamentação (deixou de fazer pelo menos uma mamada por dia). 